\title{
Bat Optimization Assisted Diabetic Retinopathy Detection Framework
}

\author{
Fatema Murshid AlBalushi \\ Department of Logistics and Transport Management, \\ International Maritime College \\ Oman, Suhar OM, Oman \\ fatemamurshidalbalushi@gmail.com
}

\begin{abstract}
Diabetic retinopathy (DR) is a disease that occurs among the persons, who were affected by diabetes for a long time. At such conditions, leakage of protein and fluid from the blood vessels occur. This work intends to establish a new automated DR recognition scheme that includes stages such as "Feature extraction and Classification". At first, feature extraction is performed; where Local Vector Pattern (LVP) and spatial map based edge detection features are extracted. Further, the extracted features are subjected to the classification phase, for which Optimized Deep Convolutional Neural Network (DCNN) is deployed as the classifier. Moreover, to accomplish better accuracy, the weights of CNN are optimally selected by means of the Bat algorithm (BA). Finally, analysis is held to validate the efficacy of the proposed model over other models.
\end{abstract}

Keywords: Diabetic Retinopathy; Local Vector Pattern; DCNN; Spatial Edge; BA Approach.

\begin{tabular}{ll} 
Nomenclature \\
\hline Abbreviations & Descriptions \\
\hline AUC & Area Under Curve \\
BA & Bat algorithm \\
CAD & Computer-Aided Diagnosis \\
DR & Diabetic retinopathy \\
DCNN & Deep Convolutional Neural Network \\
LVP & Local Vector Pattern \\
MAs & Micro Aneurysms \\
NN & Neural Network \\
WP-CNN & weighted path-CNN \\
\hline
\end{tabular}

\section{Introduction}

DR is defined "as the presence of typical retinal microvascular signs in an individual with diabetes mellitus. Higher levels of glucose in blood cause the blood vessels to take up extra glucose than usual. This paves to the surplus formation of glycoprotein. The wall of the vessel becomes thick and weak, which leads to the leak of lipids, proteins, and water that results in bulging of the fovea. [6] [7]. The detection of DR in advance avoids the stoppage of visualization in humans. DR could be spotted at an earlier stage by observing the blood vessels leaks, irregularities, and so on [8] [9].

Generally, DR is characterized when the blood vessels get affected by diabetes. During the beginning stage, the retinal arteries start leaking and small hemorrhages are formed [10]. The vessels leaked from lipoproteins cause' blurred vision. Another issue in DR is owing to the development of weak blood vessels, which divide and leaks blood in the eye. Thus the images could not be projected to the brain by the retina. This also causes blindness in persons encountered with diabetes [11].

Diagnosing and taking proper treatment for DR [12] [13] is essential that need monitoring and screening at an earlier stage. In addition, improved screening leads to an early diagnosis that lessens the threat of blindness. Currently, CAD systems are exploited, which exactly offers automated diagnosis [14]. It makes a diagnosis of DR by mining the optic disc by focusing on the above-said issues in the existing 
systems [15]. Moreover, it helps the experts in taking proper decisions and thereby, leads to faster and accurate diagnosis with higher reliability [16] [17].

The main contribution of this work is to proposed a new automated DR recognition scheme that comprises stages like "Feature extraction and Classification". The paper is arranged as follows. The reviews are presented in Section 2. Section 3 describes the framework of diabetic retinopathy detection. Section 4 explains the extraction of LVP and spatial map based edge detection features. Section 5 portrays the optimization assisted DCNN classification. Section 6 discusses the outcomes and the paper is concluded by section 7 .

\section{Literature Review}

\subsection{Related Works}

In 2020, Kumar et al. [1] suggested an enhanced scheme for recognizing MA and hemorrhages, which contributed to the improvement of earlier DR detection. In addition, an enhanced segmentation scheme was exploited in this work for segmenting the blood vessels as well as optic disc. Moreover, classification was carried out by means of the NN model, in which training was performed using the MA features. In the end, the outcomes have proved the development of the adopted model in terms of precision, accuracy, and so on.

In 2020, Zago et al. [2] implemented a novel model for identifying DR via a deep patch oriented model. The implemented detection model has lessened the complexities and it also enhanced the performance of the system using the CNN model. In addition, the betterment of the established scheme was carried out and the finer outcomes were attained for accuracy and sensitivity.

In 2019, Liu et al. [3] implemented a new technique termed as WP-CNN that exploited various weight paths of CNN. The adopted method was simulated by an ensemble learning technique. As per this model, numerous path weight coefficients were chosen optimally by means of backpropagation, and as a result, the mean of output features was considered for speedy convergence and redundancy minimization. In the end, the efficacy of the implemented scheme was established from the simulation outcomes in terms of F-measure, accuracy, and so on.

In 2018, Wan et al. [4] presented an automated method for classifying a specific set of fundus images. Accordingly, in the proposed work, the CNN approach was used for identifying DR that included segmentation, classification, and detection phases. Eventually, the enhancement of the adopted model was proven over the conventional techniques in terms of optimal accuracy.

In 2019, Shanthi and Sabeenian [5] suggested a scheme that concerned with the categorization of fundus images using the CNN framework. The CNN framework has classified the images based on the harshness of the syndrome, by which higher accuracy was obtained. In the end, the classification accuracy of the presented scheme was validated from the simulated analysis.

\section{Framework of Diabetic Retinopathy Detection}

\subsection{Proposed Architecture}

The proposed DR detection framework includes two major phases namely, feature extraction and classification. Fig. 1 demonstrates the illustration of the DR detection model. Initially, the input image I is subjected to the feature extraction phase, where LVP as well as spatial edge-based features are extracted. The subsequent phase is classification, which detects the presence or absence of the disease. For classification purposes, this work deploys the DCNN framework, in which the weights are optimally chosen using the DCNN model. The Optimized DCNN ensures the accurate detection of the disease since the weights are fine-tuned optimally. For this optimal tuning, the BA approach is deployed in this work. 

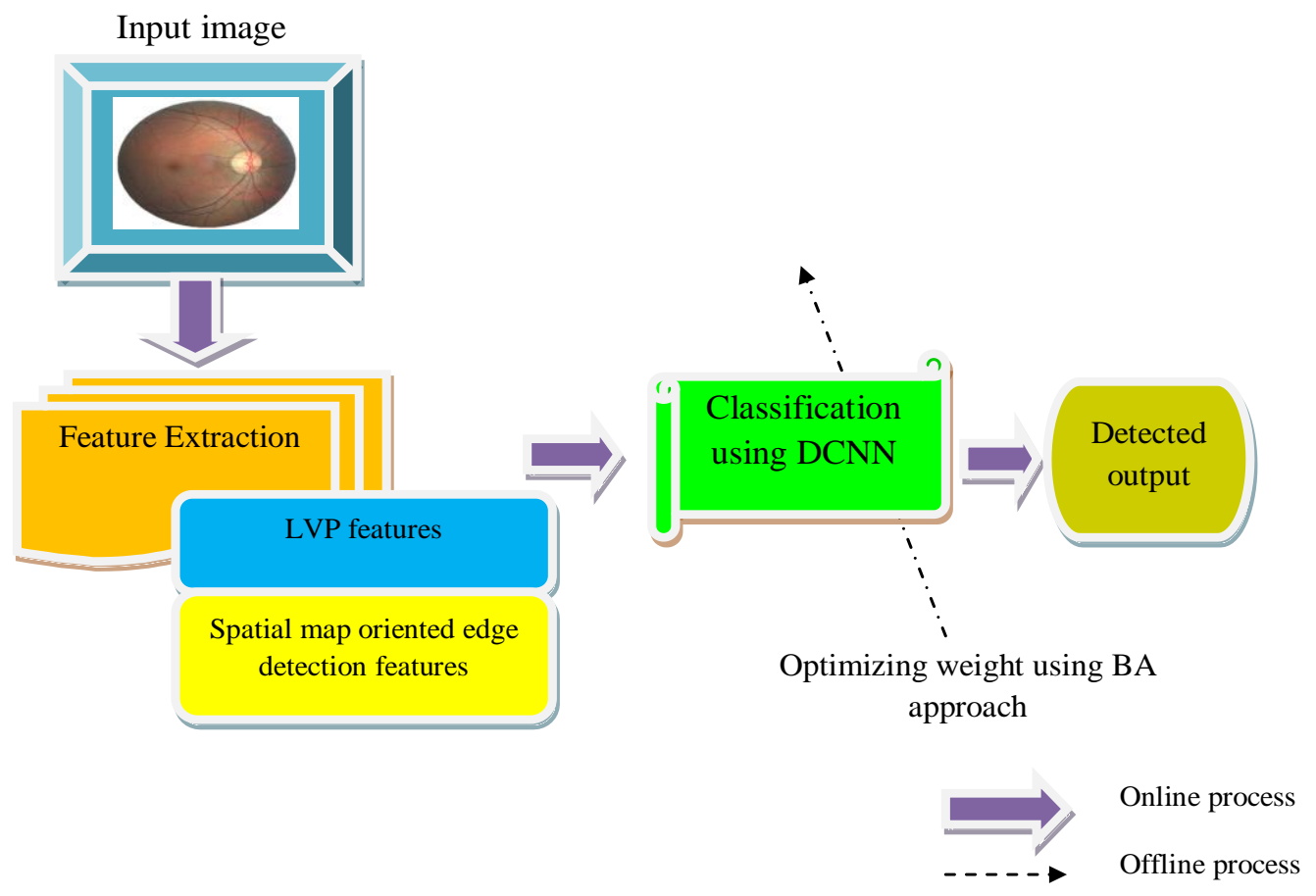

Fig. 1. Overall model of the DR detection framework

\section{Extraction of LVP and Spatial Map-based Edge Detection Features}

\subsection{Feature Extraction}

LVP: "The LVP descriptor was developed to provide various 2-D spatial structures of micropatterns with various pair-wise directions of the vector of the referenced pixel and its neighborhood". LVP [13] involves the following procedures: Considering a local sub-region $\mathrm{R}$, the vectors' direction value at reference pixel $\mathrm{O}_{\mathrm{c}}$ is indicated by $\mathrm{VE}_{\varpi, \mathrm{DI}}\left(\mathrm{O}_{\mathrm{c}}\right)$ and it is computed as shown in Eq. (1). In Eq. (1), $\mathrm{J}$ signifies the count of nearer pixels of reference pixel, $U$ indicates the radius, DI point at the distance amongst the reference pixel, and its close by pixels with $\varpi$ direction, in which $\beta$ point out the index angle of varying directions.

$$
\mathrm{VE}_{\varpi, \mathrm{DI}}\left(\mathrm{O}_{\mathrm{c}}\right)=\left(\mathrm{R}\left(\mathrm{O}_{\varpi, \mathrm{DI}}\right)-\mathrm{R}\left(\mathrm{O}_{\mathrm{c}}\right)\right)
$$

Furthermore, the $\mathrm{LVP}_{,} \mathrm{LVP}_{\mathrm{J}, \mathrm{U}, \mathrm{w}}\left(\mathrm{O}_{\mathrm{c}}\right)$ in $\beta$ direction of vector at $\mathrm{O}_{\mathrm{c}}$ is encoded, and eventually, it is determined as the series of 4 eight-bit binary patterns as shown in Eq. (2).

$$
\mathrm{F}^{\mathrm{LVP}}=\operatorname{LVP}_{\mathrm{J}, \mathrm{U}}\left(\mathrm{O}_{\mathrm{c}}\right)=\left\{\operatorname{LVP}_{\mathrm{J}, \mathrm{U}, \varpi}\left(\mathrm{O}_{\mathrm{c}}\right) \mid \varpi=0^{\circ}, 45^{\circ}, 90^{\circ}, 135^{\circ}\right\}
$$

The features extracted by means of LVP are indicated by $\mathrm{F}^{\mathrm{LVP}}$.

\subsection{Spatial Map-based Edge detection}

The spatial map oriented edge detection features permits to make use of the inherent structures in edge patches and it is found to be an effectual and computationally competent technique [14]. The features extracted using spatial map based edge detection processes are pointed out by $\mathrm{F}^{\mathrm{ED}}$.

These extracted features $\left(\mathrm{F}^{\mathrm{ED}}+\mathrm{F}^{\mathrm{LVP}}\right)$ totally denoted by $\mathrm{F}=\left(\mathrm{F}^{\mathrm{ED}}+\mathrm{F}^{\mathrm{LVP}}\right)$ are provided as input to the DCNN model for classification purposes.

\section{Optimization Assisted Deep CNN Classification for Diabetic Detection}

\subsection{Optimized DCNN Framework}

DCNN [15] is a well-known deep learning model, which encompasses three diverse layers, "convolutional layer, pooling layer, and fully-connected layers". Each neuron in the feature map is related to the adjacent neurons in the preceding layer. At the location $(a, b)$ in the $1^{\text {th }}$ layer of the corresponding $c^{\text {th }}$ 
feature map, the feature values are computed as shown by Eq. (3), where, $\mathrm{W}_{\mathrm{c}}^{1}$ refer to the weight that is fine-tuned in an optimal manner using the BA approach and $\mathrm{B}_{\mathrm{c}}^{1}$ refer to the bias term of $\mathrm{c}^{\text {th }}$ filter related to $1^{\text {th }}$ layer.

$$
Z_{a, b, c}^{1}=W_{c}^{1^{T}} V_{a, b}^{1}+B_{c}^{1}
$$

In addition, at the central location $(\mathrm{a}, \mathrm{b})$ of $1^{\text {th }}$ layer, the patched input is referred by $\mathrm{V}_{\mathrm{a}, \mathrm{b}}^{1}$.

Consequently, the activation function establishes non-linearity that helps in detecting the nonlinear features in multi-layer networks. The activation value $\left(\operatorname{act}_{\mathrm{a}, \mathrm{b}, \mathrm{c}}^{1}\right)$ corresponding to the convolutional features $Z_{a, b, c}^{1}$ is computed as per Eq. (4).

$$
\operatorname{act}_{\mathrm{a}, \mathrm{b}, \mathrm{c}}^{1}=\operatorname{act}\left(\mathrm{Z}_{\mathrm{a}, \mathrm{b}, \mathrm{c}}^{1}\right)
$$

Pooling layer: "Pooling layers in the DCNN perform the downsampling operations with the outcomes acquired from the convolutional layers". For all pooling functions pool( $\bullet$ ) corresponding to act ${ }_{\mathrm{m}, \mathrm{k}, \mathrm{c}}^{1}$, the value of $\mathrm{U}_{\mathrm{a}, \mathrm{b}, \mathrm{c}}^{1}$ is determined as exposed in Eq. (5).

$$
\mathrm{U}_{\mathrm{a}, \mathrm{b}, \mathrm{c}}^{1}=\operatorname{poo}\left(\mathrm{act}_{\mathrm{m}, \mathrm{k}, \mathrm{c}}^{1}\right), \forall(\mathrm{m} \cdot \mathrm{k}) \in \mathrm{R}_{\mathrm{a}, \mathrm{b}}
$$

The classification takes place at the final layer of DCNN, which is considered as the output layer. The loss of DCNN is pointed out by Loss and it is computed as per Eq. (6).

$$
\text { Loss }=\frac{1}{\mathrm{~K}} \sum_{\mathrm{k}=1}^{\mathrm{K}} 1\left(\theta ; \mathrm{U}^{(\mathrm{k})}, \mathrm{O}^{(\mathrm{k})}\right)
$$

The overall parameter associated with $\mathrm{W}_{\mathrm{c}}^{1}$ and $\mathrm{B}_{\mathrm{c}}^{1}$ is signified by $\theta$. Here exists $K$ count of inputoutput relations $\left\{\left(\mathrm{V}^{(\mathrm{k})}, \mathrm{U}^{(\mathrm{k})}\right) ; \mathrm{k} \in[1, \cdots, \mathrm{K}]\right\}$. The $\mathrm{k}^{\text {th }}$ input feature, the related target labels, and the output of DCNN are pointed out by $\mathrm{V}^{(\mathrm{k})}, \mathrm{U}^{(\mathrm{k})}$ and $\mathrm{O}^{(\mathrm{k})}$, in that order.

\section{Determination of Optimal Weight using the BA model}

\subsection{Solution Encoding}

The presented work aims to accomplish the accurate prediction of DR, for which, the weights of DCNN denoted by $\mathrm{W}$ is tuned optimally. For optimization purposes, the BA model is deployed. The input solution given to BA is illustrated in Fig. 2, where Nu refers to the total count of weights. Further, the objective function $(\mathrm{Obj})$ defined in the work is stated in Eq. (7), where Loss signify the error among actual and predicted result.

$$
\operatorname{Obj}=\operatorname{Min}(\text { Loss })
$$

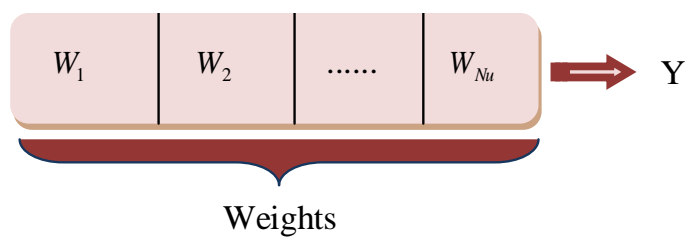

Fig. 2. Solution encoding

\subsection{BAT Algorithm}

The phases in the Bat algorithm include "initialization, evaluation, movement of virtual bats, loudness and pulse rate-based movement and termination".

Initialization: The first phase is an initialization, where the $\mathrm{n}$ bats are arbitrarily chosen and the bat's positions are initialized within search space. In addition, the constraints such as rate of the pulse $r$, loudness $D$, iteration count $t$, velocity $s_{i}^{t}$, maximal and minimal frequency of $G_{\max }$ and $G_{\min }$ are also initialized randomly. The input image is specified as shown in Eq. (8), in which, $y_{i}^{m n}$ denotes $i^{\text {th }}$ position of the input image with $\mathrm{n}$ columns and m rows. 


$$
Y_{i}=\left\{\begin{array}{ll}
y_{i}^{m n} & 0 \leq m \leq q \\
& 0 \leq n \leq f_{n}
\end{array}\right\}
$$

Evaluation: After carrying out initialization, all bats are computed with the fitness function that focuses on the minimization of loss. If the evaluated fitness is better, that value is considered as an optimal one $\mathrm{y}_{\mathrm{b}}$.

Movement of bats: After evaluation, the position of all maps are updated by means of both velocity and frequency. The formulation of the BAT algorithm is modelled as shown in Eq. (9), in which $\mathrm{G}_{\mathrm{i}}$ refers to the frequency of an image and $\gamma$ refers to the random integer.

$$
\mathrm{G}_{\mathrm{i}}=\mathrm{G}_{\min }+\left(\mathrm{G}_{\max }-\mathrm{G}_{\min }\right) * \gamma
$$

Accordingly, the image velocity is updated by means of both best values of frequency $\mathrm{G}_{\mathrm{i}}$ that are expressed by Eq. (10) and Eq. (11). In addition, $y_{i}^{t-1}$ refers to the prior iteration, $y_{b}$ points out the best value, which is considered as fitness value $\alpha$.

$$
\begin{aligned}
& \mathrm{s}_{\mathrm{i}}^{\mathrm{t}}=\mathrm{s}_{\mathrm{i}}^{\mathrm{t}-1}+\mathrm{G}_{\mathrm{i}}\left(\mathrm{y}_{\mathrm{i}}^{\mathrm{t}-1}-\mathrm{y}_{\mathrm{b}}\right) \\
& \mathrm{y}_{\mathrm{i}}^{\mathrm{t}-1}=\mathrm{y}_{\mathrm{i}}^{\mathrm{t}}-\mathrm{s}_{\mathrm{i}}^{\mathrm{t}}
\end{aligned}
$$

Loudness and pulse rate movement: At this phase, the arbitrary value of $\gamma$ is evaluated with pulse rate denoted by $r$. If the arbitrary value is better than the $r$, the novel local solutions are produced, which depends on the optimal value $y_{b}$. In addition, if the arbitrary value is lesser than the loudness, then the arbitrary value is produced. One again, the evaluation occurs among loudness and an arbitrary value. If the arbitrary solution is superior to the optimal solution, the pulse rate and loudness are updated.

Termination: The aforesaid process is continued until the entire virtual bats are updated. After the completion of iteration, the output is regarded as the optimal one.

\section{Results and discussion}

\subsection{Simulation Procedure}

The adopted DR diagnosis model was implemented in MATLAB and the results were recorded. Here, the analysis was carried out by comparing the adopted classification model over conventional classification schemes such as AlexNet, VggNet-s, VggNet-16, VggNet-19, and Google Net. Here, the analysis was carried out in terms of various measures namely, "accuracy, sensitivity, specificity, and AUC".

\subsection{Classification Analysis}

The classification analysis of the DR recognition model is exposed in Fig. 3 with respect to AUC measure. From the analysis, the ResNet has attained better AUC performance when evaluated over the other existing constraints. Particularly from Fig. 3(a), the values of AUC attained by the ResNet is $3.74 \%$, $4.62 \%, 49.96 \%, 4.13 \%$ and $6.58 \%$ better than the values attained by AlexNet, VggNet-s, VggNet-16, VggNet-19 and GoogleNet. As a result, the superiority of the BA + DCNN has been established via AUC measure. In addition, Table 1 shows the classification analysis accomplished with random constraints. On observing the attained values, the GoogleNet has attained a higher accuracy of $86.35 \%$, whereas the compared schemes have achieved comparatively minimal values. Thus, the superiority of the BA + DCNN method is proved over other traditional models. 


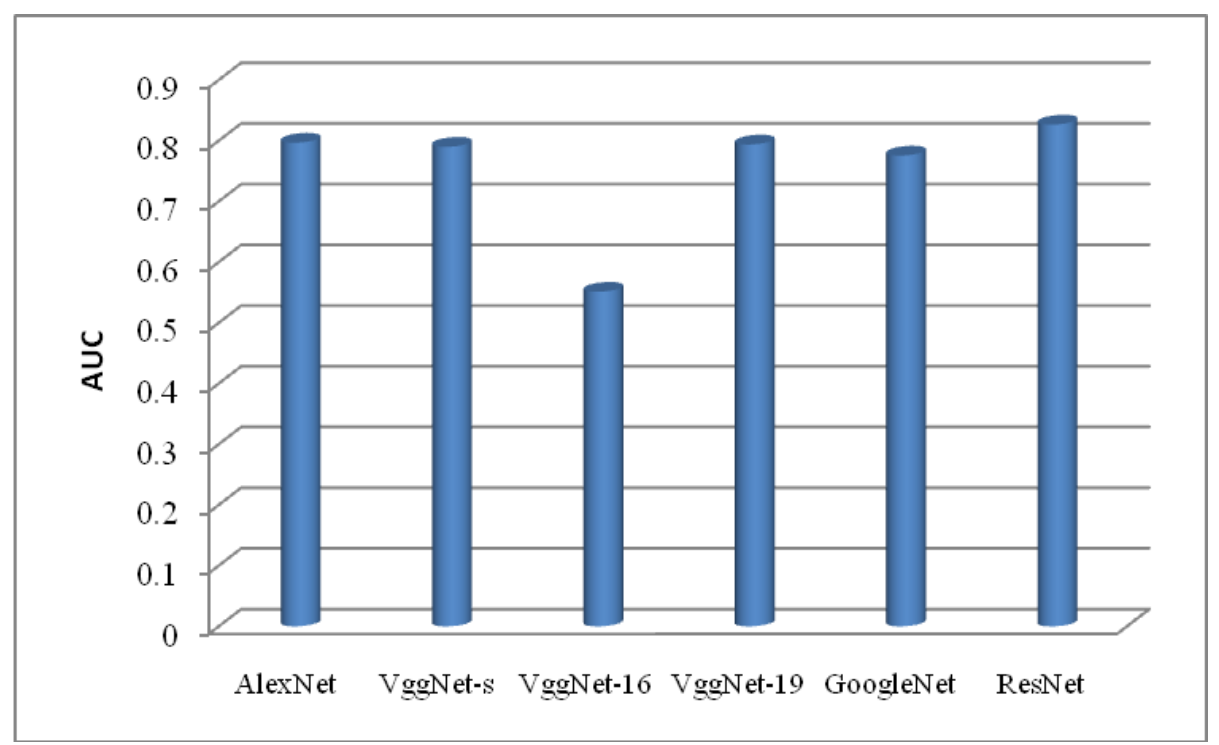

Fig. 3. Classification outcomes with arbitrarily initialized constraints with respect to AUC

Table 1: Classification outcomes attained with randomly initialized constraints

\begin{tabular}{ccccr}
\hline Model & Sensitivity & Accuracy & Specificity & \multicolumn{1}{c|}{ AUC } \\
\hline AlexNet & $39.12 \%$ & $73.04 \%$ & $90.07 \%$ & 0.7968 \\
VggNet-16 & $86.37 \%$ & $48.13 \%$ & $29.09 \%$ & 0.5512 \\
VggNet-s & $33.43 \%$ & $73.66 \%$ & $93.98 \%$ & 0.7901 \\
GoogleNet & $64.83 \%$ & $86.35 \%$ & $86.84 \%$ & 0.7756 \\
VggNet-19 & $54.51 \%$ & $82.17 \%$ & $96.05 \%$ & 0.7938 \\
ResNet & $73.77 \%$ & $0.7868 \%$ & $90.53 \%$ & 0.8266 \\
\hline
\end{tabular}

\subsection{Analysis with Hyperparameter-Tuning}

The classification analysis with hyperparameter tuning is revealed in Table II with respect to various measures like sensitivity, specificity, accuracy, and AUC. From the analysis, the VggNet-s model has attained better accuracy when compared to other models. In addition, the specificity accomplished by the VggNet-s model has revealed higher values, when evaluated over the other models.

Table 2: Classification outcomes attained with Hyperparameter-Tuning

\begin{tabular}{ccccc}
\hline Model & Sensitivity & AUC & Accuracy & Specificity \\
\hline AlexNet & $81.27 \%$ & 0.9342 & $89.75 \%$ & $94.07 \%$ \\
VggNet-19 & $89.31 \%$ & 0.9684 & $93.73 \%$ & $96.49 \%$ \\
VggNet-16 & $90.78 \%$ & 0.9616 & $93.17 \%$ & $94.32 \%$ \\
VggNet-s & $86.47 \%$ & 0.9786 & $95.68 \%$ & $97.43 \%$ \\
GoogleNet & $77.66 \%$ & 0.9272 & $93.36 \%$ & $93.45 \%$ \\
ResNet & $88.78 \%$ & 0.9365 & $90.40 \%$ & $95.56 \%$ \\
\hline
\end{tabular}

\section{Conclusion}

This paper has developed a new DR model, which included stages like feature extraction, and classification. Initially, feature extraction was carried out, by which the LVP and spatial map based edge detection features were extracted. The extracted features were then classified using the CNN model, where the weights were fine-tuned using the BA approach. Finally, the analysis was held for proving the superiority of the BA + DCNN model. Particularly on observing the AUC measure, the values attained by ResNet were $3.74 \%, 4.62 \%, 49.96 \%, 4.13 \%$, and $6.58 \%$ better than the values attained by AlexNet, VggNet-s, VggNet-16, VggNet-19, and GoogleNet. Thus, the enhancement of the BA+DCNN algorithm was validated effectively.

\section{References}

[1] T. Shanthi, R. S. Sabeenian, "Modified Alexnet architecture for classification of diabetic retinopathy images", Computers \& Electrical Engineering, vol. 76, pp. 56-64, June 2019. 
[2] S. Kumar, A. Adarsh, B. Kumar, A. K. Singh, "An automated early diabetic retinopathy detection through improved blood vessel and optic disc segmentation”, Optics \& Laser Technology, vol. 121, January 2020, Article 105815.

[3] G.T. Zago, R.V. Andreão, B. Dorizzi, E. O.T. Salles, "Diabetic retinopathy detection using red lesion localization and convolutional neural networks", Computers in Biology and Medicine, vol. 116, January 2020, Article 103537.

[4] Y.-P. Liu, Z. Li, C. Xu, J. Li, R. Liang, "Referable diabetic retinopathy identification from eye fundus images with weighted path for convolutional neural network", Artificial Intelligence in Medicine, vol.99, August 2019, Article 101694.

[5] S. Wan, Y. Liang, Y. Zhang, "Deep convolutional neural networks for diabetic retinopathy detection by image classification", Computers \& Electrical Engineering, vol. 72, pp. 274-282, November 2018.

[6] G.Leontidis, "A new unified framework for the early detection of the progression to diabetic retinopathy from fundus images", Computers in Biology and Medicine, vol. 90, pp. 98-115, 1 November 2017.

[7] M. E. G. Arias, D. Marin, B. Ponte, F. Alvarez, J. M. Bravo, "A tool for automated diabetic retinopathy prescreening based on retinal image computer analysis”, Computers in Biology and Medicine, vol. 88, pp. 100-109, 1 September 2017.

[8] R.R. Romero, J.M. Carballido, J.H. Capistrán, L. J. U. Valencia, "A method to assist in the diagnosis of early diabetic retinopathy: Image processing applied to detection of microaneurysms in fundus images", Computerized Medical Imaging and Graphics, vol. 44, pp. 41-53, September 2015.

[9] G. Gupta, S. Kulasekaran, K. Ram, N. Joshi, R. Gandhi, "Local characterization of neovascularization and identification of proliferative diabetic retinopathy in retinal fundus images", Computerized Medical Imaging and Graphics, vol. 55, pp. 124-132, January 2017.

[10] E.Saleh, J. Błaszczyński, A. Moreno, A. Valls, R. Słowiński, "Learning ensemble classifiers for diabetic retinopathy assessment”, Artificial Intelligence in Medicine, vol. 85, pp. 50-63, April 2018.

[11] M. Tavakoli, R, P. Shahri, H.Pourreza, A. Mehdizadeh, M.H. .B. Toosi, "A complementary method for automated detection of microaneurysms in fluorescein angiography fundus images to assess diabetic retinopathy”, Pattern Recognition, vol. 46, no. 10, pp. 2740-2753, October 2013.

[12] T. C. Gräsbeck, S. V. Gräsbeck, P. J. Miettinen, P. A. Summanen, "Fundus Photography as a Screening Method for Diabetic Retinopathy in Children With Type 1 Diabetes: Outcome of the Initial Photography", American Journal of Ophthalmology, vol. 169, pp. 227-234, September 2016.

[13] Kuo-Chin Fan and Tsung-Yung Hung, "A Novel Local Pattern Descriptor-Local Vector Pattern in High-Order Derivative Space for Face Recognition", Ieee Transactions On Image Processing, vol. 23, no. 7, pp. 2877-89, July 2014.

[14] P. Dollár and C. L. Zitnick, "Structured Forests for Fast Edge Detection,"IEEE International Conference on Computer Vision, Sydney, NSW, 2013, pp. 1841-1848, doi: 10.1109/ICCV.2013.231.

[15] Y. LeCun, K. Kavukvuoglu, and C. Farabet, "Convolutional networks and applications in vision", In Circuits and Systems, International Symposium on, pages 253-256, 2010.

[16] Malige Gangappa,Kiran Mai C,Sammulal P,"Enhanced Crow Search Optimization Algorithm and Hybrid NNCNN Classifiers for Classification of Land Cover Images",Multimedia Research, Volume 2, Issue 3, July 2019.

[17] Nipanikar S I,Hima Deepthi V,"Enhanced Whale Optimization Algorithm and Wavelet Transform for Image Steganography",Multimedia Research, Volume 2, Issue 3, July 2019. 